\title{
Real Cockpit Proposal for Flight Simulation with Airbus A32x Models: An Overview Description
}

\author{
José Carvalho ${ }^{1}{ }^{\mathrm{a}}$, André C. Mendes ${ }^{1} \mathbb{C}^{\mathrm{b}}$, Thadeu Brito ${ }^{1,2} \mathbb{C}^{\mathrm{c}}$ and José Lima ${ }^{1,3} \mathbb{C}^{\mathrm{d}}$ \\ ${ }^{1}$ Research Centre in Digitalization and Intelligent Robotics (CeDRI), Instituto Politécnico de Bragança, \\ Campus de Santa Apolónia, 5300-253 Bragança, Portugal \\ ${ }^{2}$ Faculty of Engineering of University of Porto, Porto, Portugal \\ ${ }^{3}$ INESC TEC - INESC Technology and Science, Porto, Portugal
}

Keywords: $\quad$ Flight Simulator, XPlane, Software-in-the-Loop, Hardware-in-the-Loop, Cockpit.

\begin{abstract}
This paper describes the several steps to build an elaborate flight simulator cockpit, where the hardware is designed based on Mechatronic principles and the proposed software was developed using agile methodologies to create a Cyber-Physical System (CPS). Furthermore, this research attempts to simulate the real environment from an aircraft as close as possible with a real scale developed cockpit. Based on this, the presented paper contributions include: (1) The implementation of a complex dynamic system such as a CPS, where the Mechatronic system is part of it; (2) The deployment of a scale model of an Airbus A32x aircraft (one of the most used), integrating into a mathematical model adapted to the operation of an aircraft flight simulation system, regarding the physical forces involved. This project is also used to captivate the students' motivation to the areas of technology such as electronics and programming and permits its development as a student project and thesis. Results allow validating the proposed cockpit.
\end{abstract}

\section{$1-$ INTRODUCTION}

Hardware-in-the-Loop (HIL) simulation is a technique used to develop, test, and validate embedded systems, by adding the complexity of the plant under control through a mathematical representation of all dynamic systems related to the test platform. These mathematical representations are called "plant simulation" (Gomez, 2001) that communicates with the integrated system to be tested. This technique reduces the development cost, time, and risk. These advantages have promoted HIL simulation to become a development standard in high-stress industries such as aeronautics and aerospace. (Ellis, 2012).

On the other hand, Software-in-the-Loop (SIL) is a tool that allows accurate model validation before field testing. It is described by the low-cost computer simulators combined with the reliability of hardware emulators. Furthermore, it is a solution where model accuracy and simulation speed do not compete, un-

\footnotetext{
a (1D) https://orcid.org/0000-0002-6074-8112

b (iD https://orcid.org/0000-0001-6390-1250

c (D) https://orcid.org/0000-0002-5962-0517

d(D) https://orcid.org/0000-0001-7902-1207
}

like traditional numerical simulators (Demers et al., 2007).

Nowadays, HIL and SIL simulations are commonly used to evaluate controls and algorithms, because they easily allow dynamic changes in the model, besides allowing a fast development and increasing safety (Zhang and Mi, 2011; Chudy and Rzucidlo, 2012). After all, in such specialized industries (as aeronautics and aerospace), because the necessity for real test flights is minimized, also reducing the number of peripherals involved and consequently decreasing the project's final cost (Sampaio et al., 2013). In the development of a HIL simulator, first one must implement the real-time "plant simulation", which is a mathematical representation of all the dynamics embedded in the plant system. Modern flight simulation techniques and implementations often result in many sophisticated and complex calculations that require a high level of computational power (Gomez, 2001; Gholkar et al., 2004), then the development of this virtual environment is a difficult task. The employed simulators in several areas, including aeronautics and aerospace, have improved by the technology development such as powerful processors, GPU, and I/O interface, among others. Thus, 
the idea is to use a realistic commercial flight simulator (COTS-FS) running on a standard PC, composing a low-cost HIL platform with enough computational power and storage resources. As a result, the simulators facilitate training on complex maneuvers and procedures that cannot be performed safely with the real aircraft, such as the failure of any of the motors or other also critical. The simulator increases the number of practical training hours, accelerates pilots' training, and notably decreases training costs (Casner SM, 2013).

There are some add-ons to the flight simulators, such as TrackIR from NaturalPoint manufacturer that allows to show the cockpit panels presented on the monitor depending on the pilot head position, but it also lacks realism. In this context, the main objective of this work is to build an elaborate flight simulator system, where the hardware is created based on Mechatronic policies, that is, focusing on the communication system and the mechanical parts. Indeed, the purposed software aims to be developed using agile methodologies to create a Cyber-Physical System (CPS). In this way, this project attempts to simulate the real environment from an aircraft as close as possible. Based on this, the project's contributions include: (1) The implementation of a complex dynamic system such as a CPS, where the Mechatronic system is part of it; (2) The deployment of a real scale model of an Airbus A32x aircraft, integrating into a mathematical model adapted to the operation of an aircraft flight simulation system, regarding the physical forces involved. Further it can be used by pilots to simulate the flights. The proposed cockpit is a modular approach since it is used by students to develop projects and thesis. This cockpit is able to be used by students from mechanic, electronics, computer science and programming courses. It is also an important way to motivate students to the areas of technology.

\section{RELATED WORK}

Some look at simulators as training platforms (Merk and Roessingh, 2016; Boril et al., 2015; Louali et al., 2011) either to improve pilots' skills or maintain their tactical training, if they were military. Real-time flight simulators (Louali et al., 2011) were also proposed for the pilot-military regime to complement their physical training, which allows immersion in a virtual environment, thus contributing to the development of decision-making skills. Also, if it can even be a free flight test center (Cen et al., 2015), with emphasis on the design, development, and verification of the simulation platform. To improve flight simu- lator analysis and evaluation, Project Magenta came about 20 years ago to become a very recognizable name in Flight Simulation and Pilot Training used in conjunction with a plethora of commercial software flight simulators well as stand-alone solutions.

A modular flight control strategy to display the improved command tracking performance with fault tolerance and reconfiguration capability was presented in (Khan et al., 2014). Manifold experiments were analyzed using the Microsoft Flight Simulator, FlightGear, and X-plane related to the CERTI Infrastructure run-time, demonstrating that High-Level Architecture (HLA) has been fit to perform the exact simulations in real-time (Gervais et al., 2012; Chaudron et al., 2014).

An automatic flight control system (AFCS) automatically controls the aircraft with high precision during surveillance, reconnaissance, and measurement flights. To test AFCS created for the utility aircraft STEMME S15, a high-performance motor glider, a ground test facility in the form of a HIL simulator was built and validated (Kaden et al., 2013). The role of pilot training in assessing performance in identifying and managing hazards, especially in the air and under changing conditions through an automated collaborative system for aviation safety, was explained in (Lofaro and Smith, 2012). The evaluation and characterization of flight training simulator using Microsoft Flight Simulator 2004 for integration with HIL Architecture to develop, test, and validate embedded systems was performed in (Lorains et al., 2011).

In (Adiprawita et al., 2008), HIL simulation was chosen as a solution for minimizing the effect of control system failure in field trial step in UAV development. A flight simulator Evaluation Course at QANTAS Airways was developed in (Scamps and Gibbens, 2005). A meta-analysis on flight simulation to distinguish the important characteristics associated with effectiveness in traditional training was performed in (Hays et al., 1992). A great review of the literature was classified, of which 26 experiments have been identified with sufficient information for the statistical meta-analysis.

Based on the previous authors' concepts, this article addresses a step forward in flight simulation. The project uses complex dynamic systems to create the CPS through mechatronics parts that approach the Airbus A32x aircraft model; the literature does not point to work with this aircraft model. The simulation covered in this work demonstrates all the real module's indicators, from the sensors to the mechatronic control systems. In the next sections, each component of this work will be described. 


\section{MAIN SYSTEM ARCHITECTURE}

The V-Model allows the design and development of complex mechatronic systems with an interdisciplinary method, where the VDI guideline 2206 can be applied to obtain systems that are more flexible and adaptable to the needs of users. The overall development process follows the V-Model that is shown in Figure 1 (Shuping and Ling, 2008; Fodor et al., 2019).

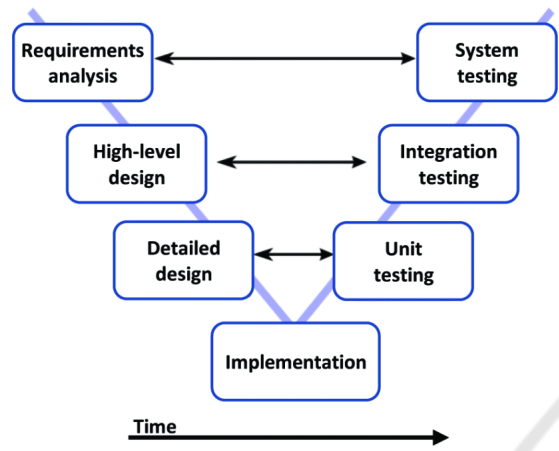

Figure 1: V-model approach used in structured systems design (Fodor et al., 2019), (Bruyninckx, 2008).

Initially all functional and non-functional requirements for the automatic flight control system are defined in the top-level specification (the developed cockpit performs the interface to the system whereas the autopilot control is provided by Toliss). They are gradually refined top-down from aircraft level via system and assembly level to the hardware and software requirements on component level. After encoding and production, the gradual integration and verification (bottom-up) follows. Each process step ends with verification tests. The final step includes validation tests with the modules mirroring the data presented in the game. In order to create a system that simulates the cockpit of the Airbus A32x model on a real scale, the system architecture shown in Figure 2 was developed. The hardware and software used in this work were developed to always communicate with each other, so the project takes the pilot in the training phase a realistic immersion during the simulation.

As is well known, there are many techniques and procedures that a fresh pilot must study to complete his/her flight license. Many of these techniques can be extensively inserted and trained in this proposed flight simulation. The entire process can be summarized through Figure 2, as well as the description of each topic below:

- Fresh Pilot: In this initial phase of training with the simulated cockpit, the pilot must already have in mind what type of flight license course,

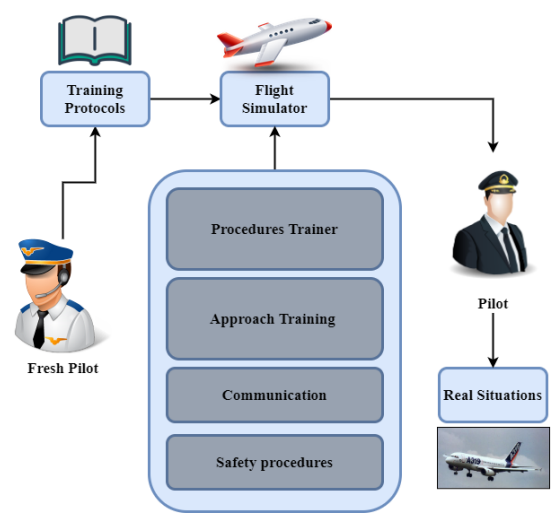

Figure 2: System Architecture.

which classifications he/she wishes to acquire, and which flights he/she intends to train.

- Training Protocols: The pilot must already have a training plan at this stage because the license has already been chosen, classification, and flights to be trained in the simulator. So, the pilot must adapt to the norms and standards required for the chosen plan. For example, a pilot of Asian origin could perform a flight simulation under conditions and standards from Europe and without having to go training in European territory.

- Flight Simulator: This part is when the flight simulation happens, that is, when the fresh pilot trains his/her skills in the simulated cockpit. The aspiring pilot will find all the modules similar to those found in the real aircraft, from the seats, buttons, indicators, and other elements present in an Airbus A32x cockpit. Licensed pilots can still maintain their degree of experience by training situations they have already experienced in real flight.

- Procedures Trainer: This tool is vital during pilots' training since the simulator is a copy almost identical to that found in the aircraft. Therefore, the pilot, together with his/her instructor, will practice possible real flight situations. Thus, the instructor will assist his/her student in a system called Flight and Navigation Procedures Trainer (FNPT), that is, to carry out training that includes necessary procedures for navigation, classification and reading of instruments, and malfunctions procedures.

- Approach Training: This training can be implemented for pilots who wish to approach different runway types but in a simulated environment. In this way, the pilot can choose from a list of options which airport he/she wants to perform the Approach Training. In this simulator, the visual 
system implemented makes a reproduction of the runway markings, runway lights, approach lights, and also the Runway Aiming Points (primary and secondary).

- Communications: In addition to navigation skills, the postulant pilot must also undertake communication training. This Communication training simulates the cooperation between the crew or simulates the pilot's communication with the Radio Station Control. Thus, it avoids possible problems with interactions between people with different types of pronunciation.

- Safety Procedures: The instructor can also add safety procedures to the novice pilot's plan, such as emergency escape, fire alarm, and emergency stops.

- Pilot and Real Situations: After exhaustively conducting numerous simulations, the instructor will evaluate his/her student to start the tests in real flight situations. Thus, the beginner pilot will be able to perform better in testing his/her flight license.

\section{HARDWARE AND SOFTWARE DEVELOPMENTS}

As stated before, this work aims to develop a flight simulation platform as close as possible to the real models. In this sense, the developed cockpit implements all system interfaces that can be found in the real cockpit of an A32x. This includes all overhead panel, main panel, Glare-shield, Pedestal and side bases systems.

Six displays are implemented in the main panel: two independent Primary Flight (PFD), one for Captain and other for First Officer; two independent Navigation Displays (ND) for Captain and First Officer; and two ECAM displays the Engine / Warning Display (EWD) and and System Display (SD). The displays show the information sent by the XHSI (eXternal High-resolution Simulator Instruments) application and are controlled by the hardware implemented in the cockpit.

On the captain side, there is the PFD (Primary Flight Display) with flight parameters, such as the horizon, speed, altitude, and some extra information regarding the flight. Then there is the Navigation Display (ND) that presents a fundamental information to the navigation, such as the flight plan, way-points, Navaids, and airports. It can also include the weather information gathered from the radar and terrain info. Moreover, the screens info configuration can be ad- justed by the Electronic Flight Instrument System (EFIS) placed above the glare shield (to reduce the effects of glare) near the autopilot commands. It allows selecting several modes, such as LS, VOR, NAV, ARC, and PLAN.

At the central panel, there is some information provided by the monitors that can be extracted by the FCOM (Flight Crew Operating Manual), among the others: Primary engine indications, fuel quantity, flap and slat position; Warning and caution alerts, or memos; Synoptic diagrams of aircraft systems, and status messages; Permanent flight data.

This structure was followed faithfully, as can be seen in the results section.

\subsection{Cyber-Physical Systems}

New challenges have emerged due to the development of recent technologies, among them is the CPS. This concept refers to integrating numerous techniques, such as computing, data network, and data acquisition system through sensing. Therefore, this integration process can provide a distributed and autonomous systems. In other words, the CPS performs a friendly coupling between the cybernetic and physical components (software and hardware); the sensor systems usually do this coupling. For a CPS system to be successful, it is necessary to model each stage of integration correctly. The modeling will be a link, or a translator, between the physical and cybernetic environments.

As indicated by (Rajkumar et al., 2010), applications using CPS will have a significant impact in a wide variety of areas. The aeronautics sector would not be left out. One of the possible applications of CPS in the aeronautics sector can be seen in flight simulation situations, where the devices in the real cockpit could be modeled to show information in the simulated environment. For example, the use of pedals in a cockpit could be synchronized with the pedal in the simulated cockpit. Thus, when the pilot is training his/her flight skills and activates the pedal in the training cockpit, consequently the pedal in the graphic simulator will also be activated at the same intensity. This process could happen for all types of devices and settings that exist in the Airbus A32x cockpit, from buttons, indicators, sensors, mechanical parts, among others. For this to happen, it is necessary to do each one's modeling since (as previously mentioned). However, this work's focus is not to describe these components' modeling due to the degree of complexity and detail required to perform the CPS of this cockpit. 


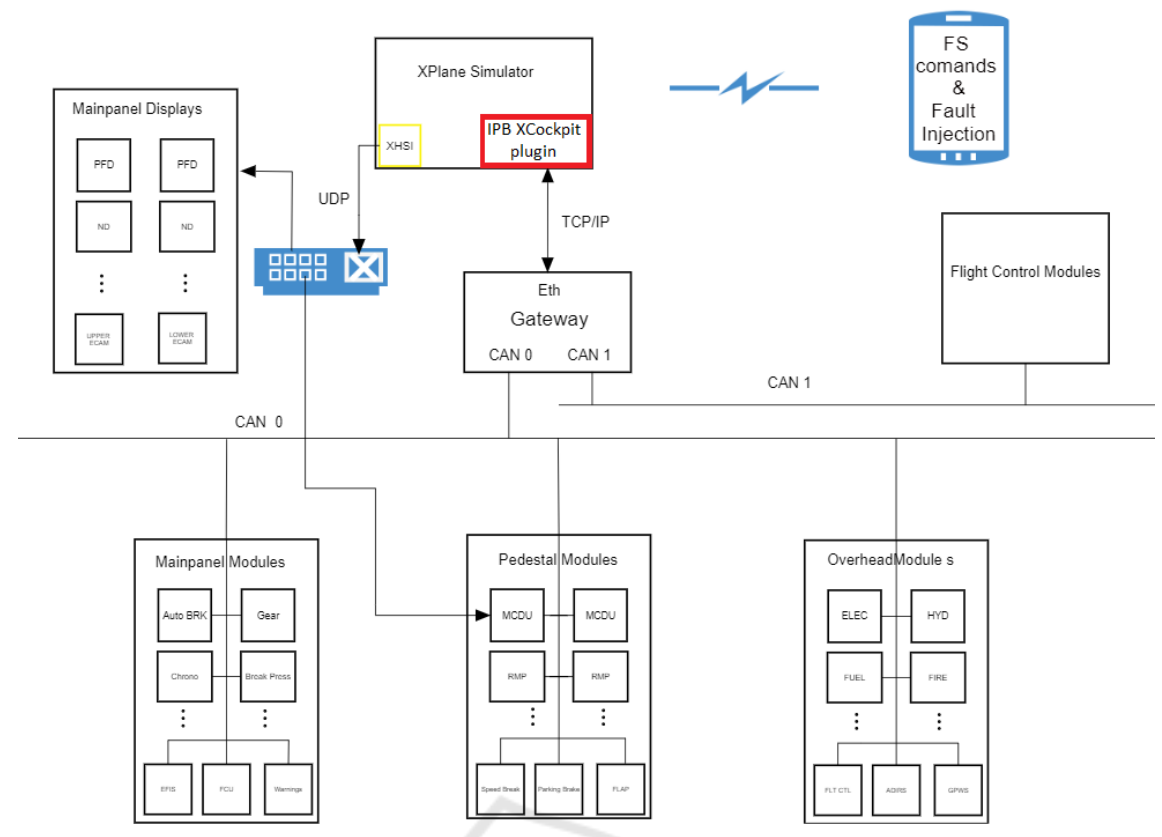

Figure 3: Main system architecture.

\subsection{Software and Hardware Components}

The proposed system is mainly composed of the $X$ - Plane Simulator setting (Meyer, 2011). Although several simulators like Microsoft's Flight Simulator and FlightGear, the $X$-Plane was chosen since it provides exceptionally accurate aerodynamic and flight models and allows for real-time data to be sent into and out of the program as well as the airfoil design. Different from the Microsoft Flight Simulator, the $X$-Plane allows input from and output to external sources. While FlightGear (Berndt, 2020) has I/O capabilities similar to $X$-Plane, it is not relatively stable and does not provide the same level of support. For all these reasons, the $X$-Plane was chosen to be used in the HIL simulator. The operation principle of $X$ - Plane is based on reading the geometric shape of any aircraft and then predicting how that aircraft will fly. The aircraft is divided into small elements, and then the forces on each element are calculated on an engineering process named "blade element theory". Based on the aircraft's mass and center of gravity, the forces are converted into accelerations, which are integrated to generate velocities and positions. All these variables are managed by a dynamics engine embedded in the game. $X$ - Plane is certified by the U.S. Agency of Aviation (FAA - Federal Aviation Administration) to train pilots. Its method ensures a reliable system since it is much more detailed, flexible, and advanced than the flight model based on stability derivatives used by most other flight simulators. The controllers developed and tested in $X$-Plane platforms have been successful when embedded in real aircraft (Garcia and Barnes, 2009) adding more credibility to the results obtained in this work. An important feature of $X-$ Plane is the capability to communicate flight data via UDP (User Datagram Protocol) connection using the IEEE754 format, as well as the ability to exchange information with the external environment.

The developed cockpit uses a plugin to communicate with clients and servers based on callback functions.

The chosen airplane model for this work is an A32x, a member of the Airbus A320 family of shortto medium-range, narrow-body, commercial passenger twin-engine jet airliners manufactured by Airbus.

The simulation environment is being executed on a desktop PC with a core I9-9900K Processor, 32Gb DDR4 memory, a Nvidia ${ }^{T M}$ GeForce ${ }^{T M}$ RTX 2080 Ti graphics card and a solid-state drive. This system is powered by a $1 \mathrm{~kW}$ power supply. All the connections to the cockpit instruments are based on Ethernet, as it can be presented by figure 3 . There are two main connections established that uses UDP and TCP to send and receive information to the developed modules. Moreover, it will further be able to receive faults injection by a mobile device.

The main panel displays (PFD's, ND's, EWD, SD and MCDU's pedestal displays) are connected by the UDP protocol using XHSI. The XHSI mod- 
ule, that is an add-on for the X-Plane flight simulator and displays the PFD, ND, ECAM (Electronic Centralized Aircraft Monitor), Flaps and Gear indicator, Clock/Chronometer, etc... sends data to the displays each one connected to a rapsberry pi. The raspberry pi accesses to the mainpanel monitor with a HDMI, VGA, DVI, and LCD Control board work for 10.4inch G104X1 L04 1024x768 LCD panel.

On the other side, the TCP connection will handle the communication to the panels instruments, such as buttons, lights, actuators among others.

The CAN/ETH gateway connects most cockpit buttons and commands and the information is sent between cockpit and simulator PC or simulator cockpit via TCP/IP, i.e. messages are confirmed by the protocol. In the case of PFD, ND, ECAM and MCDU displays on the pedestal the information is not confirmed and sent by UDP (multicast). This has everything to do with performance. It is not necessary to confirm the message because in case of error the next message refreshes the displays with correct info.

For that purpose, it was developed a plugin (IPB XCockpit, detailed on Figure 4) that sends and receives TCP packets from/to the Gateway. This gateway allows to connect the CANO and CAN1 buses where all the modules are connected.

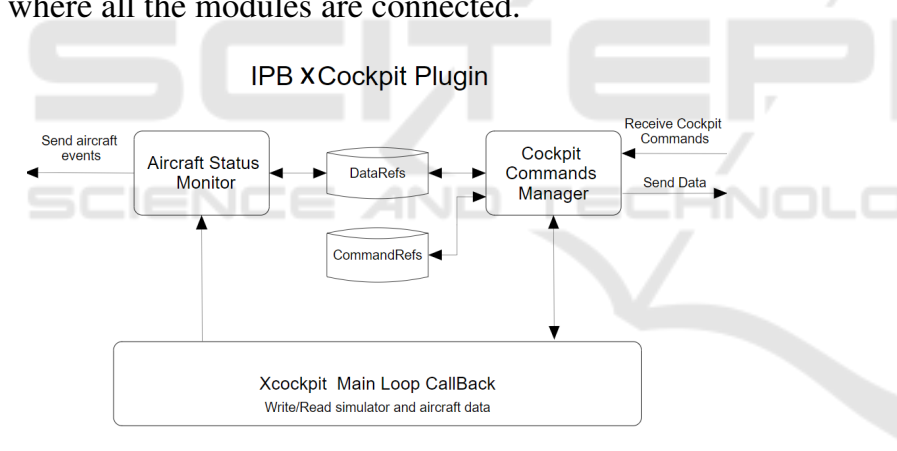

Figure 4: IPB XCockpit plugin detailed.

This developed IPB XCockpit plugin owns a main loop callback that writes and reads the simulator and aircraft data in order to feed the aircraft status monitor and receives commands from the cockpit commands manager. This information is stored into the data refs and command refs respectively. By this way, it is possible that the cockpit instruments can read the airplane variables and also control it.

\section{RESULTS}

The developed system is composed by software, hardware and mechanical components. The mechanical design of instruments and actuators is not the focus of this paper. The software is executed on a computer and communicates with a intelligent sensors network using a gateway (Ethernet and CAN bused converter). The intelligent sensor network is spread by all over the cockpit as a modular approach. Some modules of hardware and mechanical components are presented in Figures 5 and 6 . The first one shows the flight screen on a wall television, the front panel composed by the FCU (Flight Control Unit) where the Autopilot controls are placed. The Barometer system are installed on the Electronic Flight Instrument System where warnings and master caution indicators are presented also. On the other hand, the radio controls and communication system are presented on Figure 6.

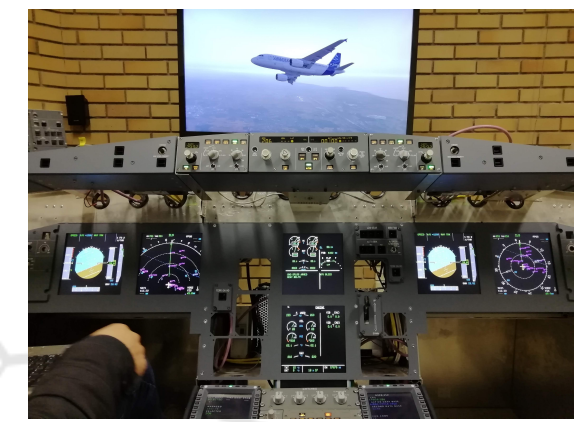

Figure 5: Under development of frontal cockpit at IPB.

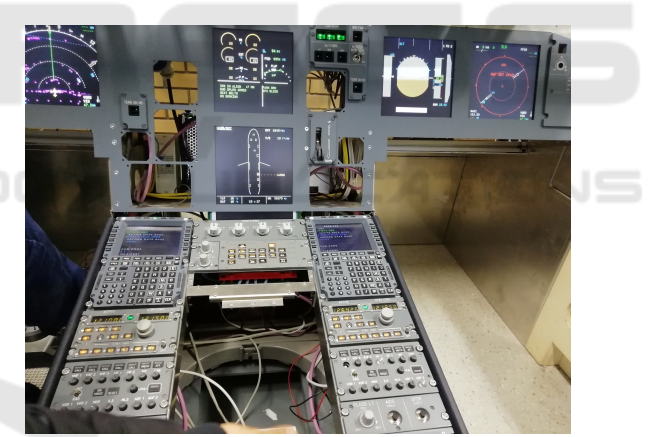

Figure 6: Under development of pedestal panel cockpit at IPB.

All these developed modules are communicating and the flight simulator assumes the variables introduced by the user on the cockpit modules. It can be validated by Figure 7 where it is possible to observe the EFIS and the FCU Flight Control Unit in which autopilots can be activated either in direct or managed mode. The BARO was configured, as example on 29.92 in $\mathrm{Hg}$. On EFIS, inside the PFD control part, it is possible to choose between $\mathrm{Hg}$ and $\mathrm{hPa}$ and using the encoder to enter the value of the barometric pressure or standard reference STD. The autopilot was configured with a speed of 207 knots, a heading of 23 degrees and an altitude of 19700 feet. As it can be observed, the instruments of the flight (on the wall television) assumed this parameters. As previ- 


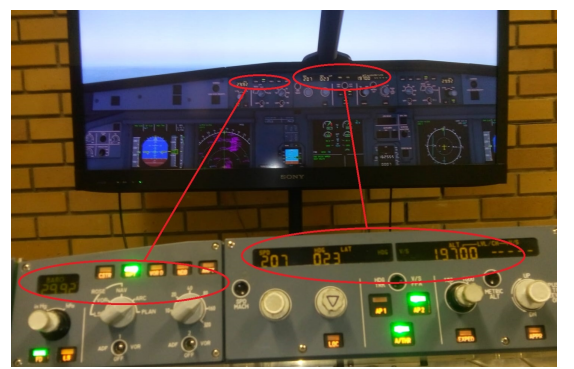

Figure 7: BARO and Autopilot control panel assuming the programmed values at the developed cockpit validating the communication between modules.

ously addressed, the monitors presented on the frontal panel, will display information as the real cockpit. Each monitor uses a Raspberry pi 4 and some examples are presented on Figure 8. Table 1 details each screen information.

Table 1: Main panel Monitors description.

\begin{tabular}{|c|c|}
\hline Monitor & Description \\
\hline \hline (a) & Door information (DOOR) \\
\hline (b) & Engine/Warning Display (E/WD) \\
\hline (c) & Engines (ENG) \\
\hline (d) & Primary Flight Display (PFD) \\
\hline (e) & Navigation Display (ND) \\
\hline
\end{tabular}

These monitors can be configured to show different information and can be selected by the Electronic Centralized Aircraft Monitor (ECAM) Control Panel. It will be possible to use the wall television to show the landscape and environment as the windows on the real cockpit. As result, it was proposed a cockpit owning several instruments, monitors and controls to make the flight simulation more realistic. All the instruments were communicating with the simulator allowing the pilot to have a flight experience more related with a real components instead of a television emulated instruments.

\section{CONCLUSIONS \& FUTURE WORK}

The presented paper described the development of a cockpit to be used for flight simulations of Airbus $\mathrm{A} 32 \mathrm{x}$. It presented the proposed mechanical and hardware components as well as its communication based on Ethernet and CAN buses. The real scale main panel of a cockpit was developed and can be used to test pilots on flight task. The instruments presented on panels, such as control buttons, lights and displays were tested in several simulated flights that validate the proposed approach. As implemented examples are the FCU (Flight Control Unit), the EFIS (Electronic Flight Instrument System), and the ECAM (Electronic Centralized Aircraft Monitor) control panel to select system pages on System Display. This modular approach had supported more than fifty students working on this project. As it can be noted, this paper presented a continuous working-inprogress development of a real cockpit to be used in simulation and there are several modules that can be included. As future work direction, it can be pointed out a projection based on three projectors using an immersive methodology and other instruments that will fit on the designed and planned, such as the development of the Throttle control system with motorized trim wheel, as well as the implementation of all overhead panels such as Ligh, Elec, HYD/Fuel, Fire, FLT CTL, ADIRS, Office and GPWS among the others.

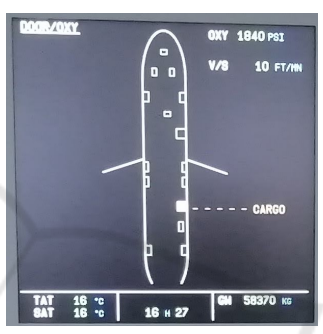

(a)

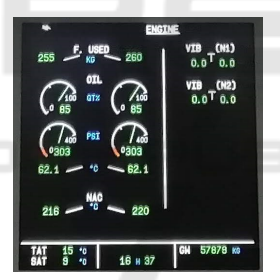

(c)

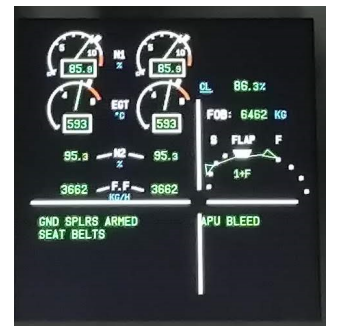

(b)

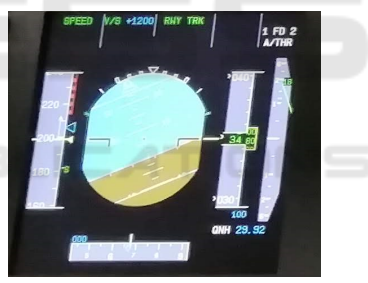

(d)

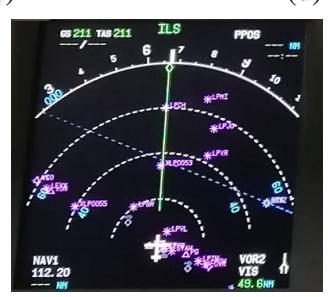

(e)

Figure 8: Monitors of the front cockpit panel. Monitors can be selected by the Electronic Centralized Aircraft Monitor (ECAM) Control Panel a) Door information (DOOR), b) Engine/Warning Display (E/WD), c) Engines (ENG), d) Primary Flight Display (PFD) where horizon, speed and altitude information is presented, e) Navigation Display (ND) with waypoints, navaids and airports localization. 


\section{ACKNOWLEDGEMENTS}

This work has been supported by FCT - Fundação para a Ciência e Tecnologia within the Project Scope: UIDB/05757/2020.

\section{REFERENCES}

Adiprawita, W., Ahmad, A., and Sembiring, J. (2008). Hardware in the loop simulator in uav rapid development life cycle. arXiv.

Berndt, J. (2020). Flightgear: an open-source flight simulator.

Boril, J., Leuchter, J., Smrz, V., and Blasch, E. (2015). Aviation simulation training in the czech air force. In 2015 IEEE/AIAA 34th Digital Avionics Systems Conference (DASC), pages 9A2-1. IEEE.

Bruyninckx, H. (2008). V model from structured systems design theory.

Casner SM, Geven RW, W. K. (2013). The Effectiveness of Airline Pilot Training for Abnormal Events. Human Factors.

Cen, F., Li, Q., Fan, L., Liu, Z., and Sun, H. (2015). Development of a pilot-in-loop real-time simulation platform for wind tunnel free-flight test. In 2015 IEEE International Conference on Information and Automation, pages 2433-2438. IEEE.

Chaudron, J.-B., Saussié, D., Siron, P., and Adelantado, M. (2014). Real-time distributed simulations in an hla framework: Application to aircraft simulation. Simulation, 90(6):627-643.

Chudy, P. and Rzucidlo, P. (2012). Hil simulation of a light aircraft flight control system. In 31th IEEE Digital Avionics Systems Conference, pages 6D111-6D112. IEEE.

Demers, S., Gopalakrishnan, P., and Kant, L. (2007). A generic solution to software-in-the-loop. Military Communications Conference 2007. MILCOM 2007.

Ellis, G. (2012). Chapter 13 - model development and verification. In Ellis, G., editor, Control System Design Guide (Fourth Edition), pages 261 - 282. Butterworth-Heinemann, Boston, fourth edition edition.

Fodor, A., Jánó, R., and Fizeșan, R. T. (2019). Characterization of the $\mathrm{v}$-model approach in thermal design process. In 2019 IEEE 25th International Symposium for Design and Technology in Electronic Packaging (SIITME), pages 367-370.

Garcia, R. and Barnes, L. (2009). Multi-uav simulator utilizing x-plane. In Selected papers from the 2 nd International Symposium on UAVs, Reno, Nevada, USA June 8-10, 2009, pages 393-406. Springer.

Gervais, C., Chaudron, J.-B., Siron, P., Leconte, R., and Saussié, D. (2012). Real-time distributed aircraft simulation through hla. In 2012 IEEE/ACM 16th International Symposium on Distributed Simulation and Real Time Applications, pages 251-254. IEEE.
Gholkar, A., Isaacs, A., and Arya, H. (2004). Hardware-inloop simulator for mini aerial vehicle. Department of Aerospace Engineering on IIT at Bombay, 2(2).

Gomez, M. (2001). Hardware-in-the-loop simulation. Embedded System Design.

Hays, R. T., Jacobs, J. W., Prince, C., and Salas, E. (1992). Flight simulator training effectiveness: A meta-analysis. Military psychology, 4(2):63-74.

Kaden, A., Boche, B., and Luckner, R. (2013). Hardwarein-the-loop flight simulator - an essential part in the development process for the automatic flight control system of a utility aircraft. In AIAA Modeling and Simulation Technologies Conference and Exhibit.

Khan, A. H., Khan, Z. H., and Khan, S. H. (2014). Optimized reconfigurable autopilot design for an aerospace cps. In Computational intelligence for decision support in cyber-physical systems, pages 381420. Springer.

Lofaro, R. J. and Smith, K. M. (2012). The aviation operational environment: Integrating a decision-making paradigm, flight simulator training and an automated cockpit display for aviation safety. In Technology Engineering and Management in Aviation: Advancements and Discoveries, pages 241-282. IGI Global.

Lorains, M., MacMahon, C., Ball, K., and Mahoney, J. (2011). Above real time training for team invasion sport skills. International Journal of Sports Science \& Coaching, 6(4):537-544.

Louali, R., Belloula, A., Djouadi, M. S., and Bouaziz, S. (2011). Real-time characterization of ms flight simulator 2004 for integration into hardware in the loop architecture. In 19th Mediterranean Conf. on Control \& Automation (MED), pages 1241-1246. IEEE.

Merk, R. and Roessingh, J. (2016). Assessing behaviour of cognitive agents in a flight simulator with fighter pilots. In 2016 IEEE International Conference on Systems, Man, and Cybernetics (SMC), pages 004842004847. IEEE.

Meyer, A. (2011). X-plane operation manual.

Rajkumar, R., Lee, I., Sha, L., and Stankovic, J. (2010). Cyber-physical systems: the next computing revolution. In Design automation conference, pages 731736. IEEE.

Sampaio, R. C. B., Becker, M., Freschi, L. W., and Montanher, M. P. (2013). Novel sil evaluation of an optimal h1 controller on the stability of a mav in flight simulator. In Aerospace Conference, pages 186D11186D12. IEEE.

Scamps, A. and Gibbens, P. (2005). Development of a flight simulator evaluation course at qantas. In AIAA Modeling and Simulation Technologies Conference and Exhibit, page 6114.

Shuping, L. and Ling, P. (2008). The research of v model in testing embedded software. In 2008 International Conference on Computer Science and Information Technology, pages 463-466.

Zhang, X. and Mi, C. (2011). Hardware-in-the-loop and software-in-the-loop testing for vehicle power management. In In proceedings of Vehicle Power Management, Power Systems. Springer. 